\title{
Evaluation of Underwater Positioning by Hanged ROV from USV
}

\author{
Soji Shimono, Hitoshi Arisumi, Shin Kato, Uichi Nishizawa, and Shigeki Toyama
}

\begin{abstract}
In this paper, the position measurement method of an underwater inspection system by a remotely operated vehicle (ROV) hanging from an unmanned surface vehicle (USV) is evaluated. Since the ROV goes directly under the USV, its underwater position can be estimated from its depth and the position of the USV. On the other hand, the horizontal position difference between both vehicles affects estimation accuracy. Thus, the position difference when the system is in a water current and when it is moving was evaluated by a simulation. As a result, in low flow rate, the difference falls within the range considered to be practical. In addition, it is confirmed by simulation and experiment that the positional difference during movement can be reduced by the moving method using both vehicles thrusters. Then, an inspection demonstration was carried out. Even in the real environment, the effect that the hanged ROV is located directly below the USV is confirmed and vertically inspection was easily performed.
\end{abstract}

Index Terms-Underwater inspection, unmanned surface water vehicle, remotely operated vehicle, underwater cable.

\section{INTRODUCTION}

Over the course of history, humans have constructed social infrastructure and civilizations have progressed. Conversely, deteriorating social infrastructure involves the risks of failing functions or serious accidents. Thus, the long-term usage of social infrastructures necessitates maintenance to avoid the fore-mentioned risks.

Rapid increases in the number of social infrastructures is a recent problem in Japan [1]. The first step in maintenance involves an inspection to understand the conditions of social infrastructures. However, implementing the overall inspection of many existing social infrastructures involves a tremendous amount of effort. Thus, efficient robotic inspection systems are required to resolve this issue.

A dam corresponds to a social infrastructure that must be inspected and Research on dam inspection by a robot also has been conducted [2], [3]. A main problem in underwater inspection relates to the difficulty in understanding the inspected position. Advanced management in future requires position information, which is very important for the database management, as well as for determining the

Manuscript received January 30, 2017; revised March 23, 2017.

Soji Shimono is with the Q.I Incorporated, 4-7, Fukuura 2-chome, kanazawa-ku, Yokohama, Japan (e-mail: shimono@qi-inc.com).

Hitoshi Arisumi and Shin Kato is with National Institute of Advanced Industrial Science and Technology (AIST), AIST Central 2, 1-1-1 Umezono, Tsukuba, Japan (e-mail: h-arisumi@aist.go.jp, shin.kato@aist.go.jp).

U. Nishizawa and S. Toyama are with the Tokyo University of Agriculture and Technology, 2-24-16, Nakacho, Koganei, Tokyo, Japan. (e-mail: n-uichi@cc.tuat.ac.jp, toyama@cc.tuat.ac.jp). periodical deterioration of social infrastructures.

It is not possible to use a Global Navigation Satellite System (GNSS) during underwater inspections. Thus, generally, an acoustic positioning system is used for underwater positioning and it is usually deployed to a support ships of underwater vehicles. However, it is difficult for these types of ships to be transported in a dam, and thus it is necessary to install the acoustic positioning device at a dam site whenever an inspection is performed. Thus, the establishment of an inspection system is complicated. Additionally, these positioning systems increase the cost of the system.

As mentioned above, a novel robotic visual inspection system that consists of an unmanned surface vehicle (USV) and a remotely operated vehicle (ROV) that hangs from the USV has been developing (Fig. 1) [4]. In this study, this inspection system corresponds to a dam inspection system, and its USV corresponds to a surface water platform (SWP) and the ROV corresponds to an underwater platform (UWP).

A feature of the dam inspection system is its positioning method. The SWP has a cable winch to hang the UWP and the UWP is designed as a negative buoyant. Thus, the UWP remains immediately beneath the SWP due to its own weight. Additionally, the depth of the UWP is determined by the length of the cable deployed from the SWP. The position of the SWP can be measured by the GNSS or other methods such as optical positioning. Therefore, the position of the UWP is estimated from the position of the SWP and depth of the UWP. The cost of the acoustic positioning system can be reduced, and it is easy to set up the inspection system at the dam site.

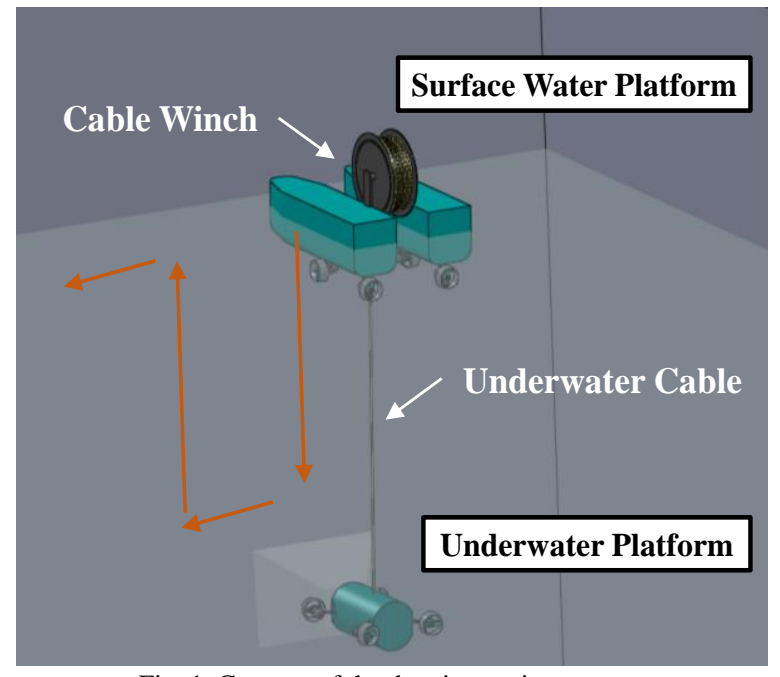

Fig. 1. Concept of the dam inspection system.

Conversely, when the dam inspection system moves or a 
water current exists at the inspection site, it is expected that a horizontal position difference occurs between the SWP and the UWP. The motivation of this study involves evaluating this difference to understand the practicalities of the proposed positioning method. The study evaluates the difference by a simulation and an experiment involving the usage of a developed test bed of the dam inspection system. Section II reports on the specifications of the developed test bed. Section III discusses the proposed dynamics model of the dam inspection system and shows the results of the simulation. Section IV describes the results of a tank test and an inspection demonstration using the test bed.

\section{DEVELOPED SYSTEM FOR DAM INSPECTION}

We now describe the developed test bed of the dam inspection system. Fig. 2 and Table I present the appearance and specifications of the test bed, respectively.

The power is supplied by the battery on the SWP, and the system is controlled through a wireless LAN communication linked to a human interface unit. Hence, the test bed is not constrained by a tether cable deployed from the land.

\section{A. Surface Water Platform}

The SWP is a twin-hulled ship. It is equipped with four $150-\mathrm{W}$ thrusters that control the surge, sway, and yaw and is installed with a cable winch that suspends the UWP. The winch can store a $100 \mathrm{~m}$ cable. The external sensor is equipped with a wide-angle camera for operation, and the distance and relative angle to the dam's wall are measured by two laser range sensors installed on both sides of the SWP. And a MEMS based GNSS aided inertial navigation system (GNSS/INS) is equipped. It consists of 3-axes accelerometer, gyroscope, magnetic field sensor, and a GNSS receiver.

\section{B. Underwater Platform}

The UWP is structured as a pressure hull enclosed with guards. The pressure hull contains an electronic device. Similarly to the SWP, the UWP is equipped with four $90-\mathrm{W}$ thrusters. It also carries a camera with a resolution of $1920 \times$ 1080 pixels, four LED lamps for underwater visual inspection, two ultrasonic range sensors that measure the distance and the relative angle to the dam's wall, and a laser marker for estimating the dimensions of cracks. And the UWP has the same GNSS/INS sensor as the SWP.

\section{System}

Fig. 3 is a block diagram of the system of the test bed. The operation command to the human interface unit (Fig. 4) is sent to the CPU unit on the SWP through the wireless LAN. In response, the CPU unit sends a control message to the main control board and the sensor I/O board on the CAN bus. According to the operation command, these boards control the thruster, cable winch, and sensors. To enable proper operation on the operation interface monitor, the system displays images of the IP cameras on the Ethernet of the test bed and the sensor information obtained from the main control board and sensor I/O board.

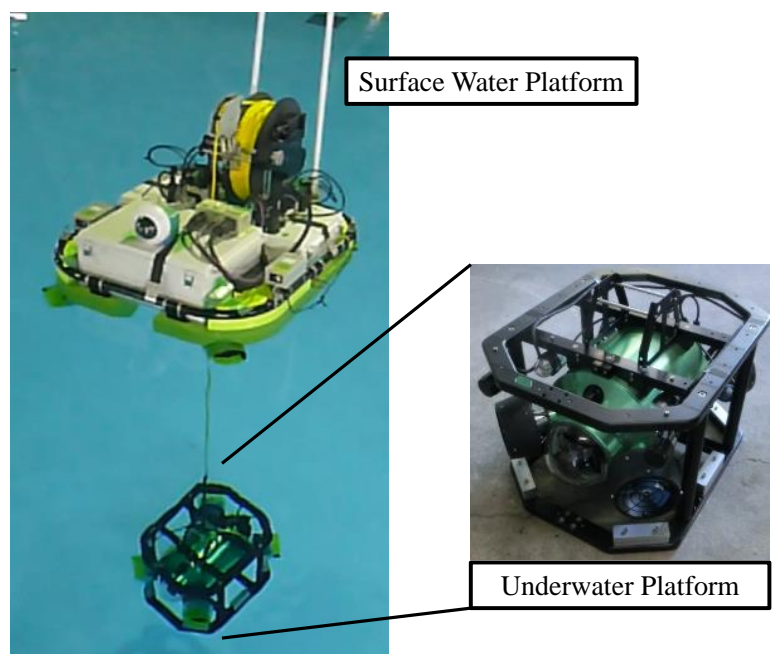

Fig. 2. Developed test bed of the dam inspection system.

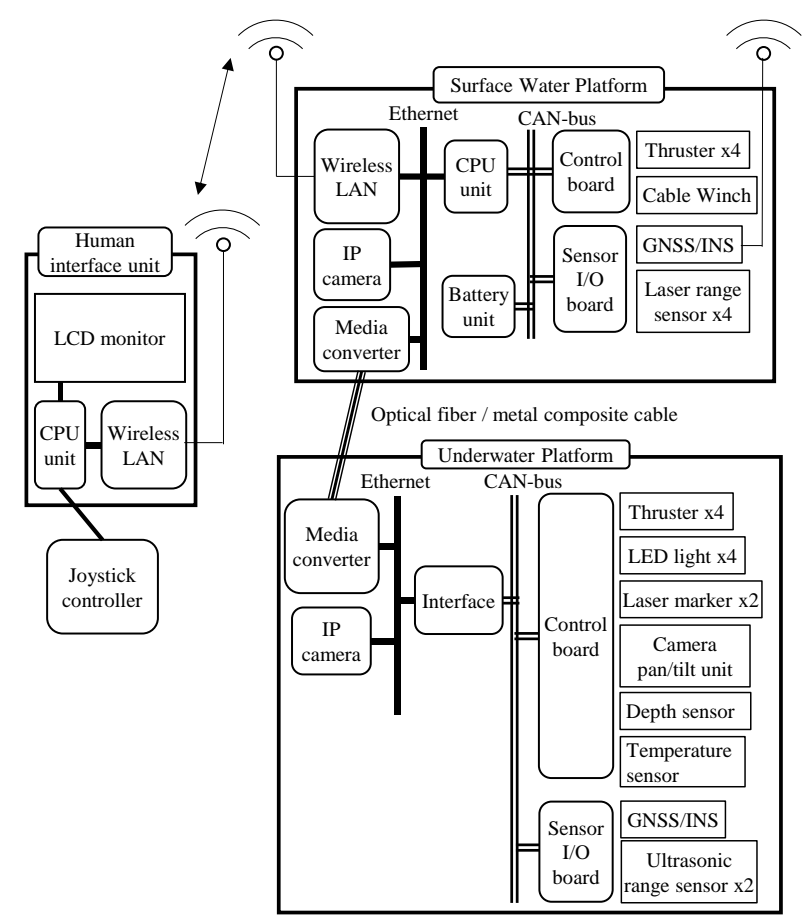

Fig. 3. System diagram of the test bed.

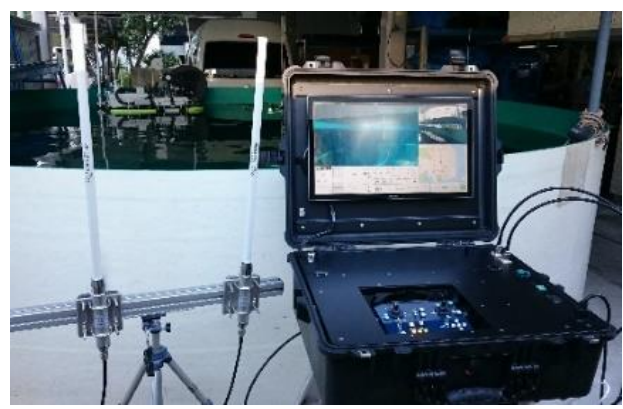

Fig. 4. Human interface unit.

TABLE I: SPECIFICATION OF THE TEST BED

\begin{tabular}{ccc}
\hline \multicolumn{3}{c}{ TABLE I: SPECIFICATION OF THE TEST BED } \\
\hline \hline SWP & UWP \\
\hline Dimension $(\mathbf{m})$ & $1.0 \times 0.85 \times 0.7$ & $0.54 \times 0.44 \times 0.35$ \\
\hline Weight $(\mathbf{k g})$ & 82 & 22.5 \\
\hline Thruster & 150W x 4 Horizontal & 90W x 4 Horizontal \\
\hline Cable Winch & 100m Cable Capacity & - \\
\hline Depth $(\mathbf{m})$ & - & 100 \\
\hline \hline
\end{tabular}




\section{Modeling AND Simulations}

The horizontal position difference between the SWP and the UWP is expected to be occurring when the dam inspection system is in motion and when it is in water current. This is effect the position estimation accuracy of the dam inspection system. In order to evaluate this position difference, a dynamics model is constructed and a simulation is implemented.

\section{A. Modeling of the Dam Inspection System}

The main motion pattern during the dam inspection is assumed to occur along the surface of the dam wall. The model was designed on a two-dimensional plane of the heave and sway direction of the dam inspection system.

Underwater cables have been modeled in studies of underwater towing systems, ROVs [5], [6]. In these models, the cable is expressed as a series of cable elements of specified length. We adopt the same approach, regarding the cable elements as weightless rigid rods with point masses at each joint. Each point mass is the origin of a cable element coordinate system. Furthermore, we assume that the connecting point transmits force but not the moment.

Four coordinate systems are defined: a space-fixed coordinate system $O_{o}-X_{o} Y_{o}$ with the water surface as the zero point on the $Y_{o}$-axis, an SWP-fixed coordinate system $O_{b}-X_{b} Y_{b}$, a cable element fixed coordinate system $O_{c i}-X_{c i} Y_{c i}$ (where $i$ is the number of the cable element), and a UWP-fixed coordinate system $O_{s}-X_{s} Y_{s}$ (Fig. 5).

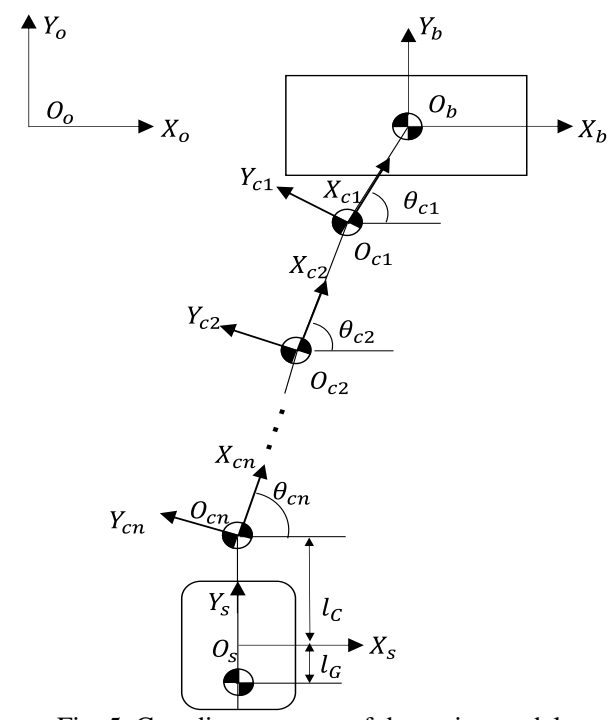

Fig. 5. Coordinate system of dynamics model.

The SWP is modeled as a rigid body. The origin of the SWP coordinate system and the cable connecting point are set to the center of mass. The UWP is also modeled as a rigid body, and the origin of its coordinate system is the center of buoyancy. The center of mass and cable connecting points are set at lengths $l_{G}$ lower and $l_{C}$ higher, respectively rather than the center of buoyant force on the $Y_{s}$.

In addition to the forces between the elements, which are connected, we consider the fluid resistance $F_{V}$, the added mass effect $F_{A}$, the buoyant force $F_{B}$, and gravity $F_{G}$. The fluid resistance and added mass effect act on the origin of each coordinate frame. The fluid resistances along the local $x$ and $y$ axes are given by (1) and (2), respectively; the corresponding added mass effects are given by (3) and (4).

$$
\begin{gathered}
F_{V x}=-(1 / 2) \rho S_{x} C d_{x} \dot{x}|\dot{x}| \\
F_{V y}=-(1 / 2) \rho S_{y} C d_{y} \dot{y}|\dot{y}| \\
F_{A x}=-m_{a x} \ddot{x} \\
F_{A y}=-m_{a y} \ddot{y}
\end{gathered}
$$

where the variables $S, C d$, and $m_{a}$ denote the area, fluid drag coefficient, and added mass, respectively. The subscripts $x$ and $y$ refer to the corresponding axes.

Gravity acts on each center of mass in the $Y_{o}$-axis direction; similarly, the buoyant force acts on each coordinate origin in the $Y_{o}$-axis direction. The cable buoyancy is ignored. The gravitational force is given by (5); buoyant forces on the SWP and UWP are calculated by (6) and (7),

$$
\begin{gathered}
F_{G}=-m g \\
F_{B b}=\rho S_{y} y_{b} g \\
F_{B s}=\left(m-W_{a q}\right) g
\end{gathered}
$$

where $\rho, y_{b}, W_{a q}$, denote the water density, the position in the $y$ direction of the $O_{b}-X_{b} Y_{b}$ viewed from $O_{o}-X_{o} Y_{o}$, the weight in water of the UWP, respectively. This model is constructed on Wolfram System Modeler software.

\section{B. Identification of the Fluid Parameters of the Test Bed}

The fluid parameters of the test bed were estimated by a step response test in the pool to compare the motion between the test beds to verify the model. The test was conducted independently for the SWP and UWP. The change in position was recorded when the step force was attached. The added mass and the fluid coefficient are obtained by the least

\begin{tabular}{|c|c|c|c|}
\hline & SWP & UWP & Cable element \\
\hline$m(\mathbf{k g})$ & 82 & $\begin{array}{l}22.5\left(W_{a q}=1.7\right) \\
24.5\left(W_{a q}=3.5\right)\end{array}$ & $0.048 \times L / n$ \\
\hline Inertia $\left(\mathrm{kg} \mathrm{m}^{2}\right)$ & 6.2 & 0.6 & 一 \\
\hline Length (m) & - & - & $L / n$ \\
\hline$S_{x}\left(\mathbf{m}^{2}\right)$ & 0.21 & 0.19 & $7.7 \times 10^{-3} \times L / n$ \\
\hline$S_{y}\left(\mathbf{m}^{2}\right)$ & 0.61 & 0.24 & $46.6 \times 10^{-6}$ \\
\hline$C d_{x}$ & 2.11 & 1.73 & 0.9 \\
\hline$C d_{y}$ & 1.7 & 1.45 & 1 \\
\hline$m_{a x}(\mathbf{k g})$ & 166.2 & 98.0 & $46.6 \times 10^{-3} \times L / n$ \\
\hline$m_{a y}(\mathbf{k g})$ & 100 & 100 & 0 \\
\hline$l_{C}(\mathbf{m})$ & 一 & 0.275 & 一 \\
\hline$l_{G}(\mathbf{m})$ & - & 0.2 & 一 \\
\hline$\rho\left(\mathrm{kg} / \mathrm{m}^{3}\right)$ & & 1000 & \\
\hline
\end{tabular}
squares method, and this fits the displacement obtained from the double integration of acceleration $a$ of the equation (8) to the recorded change in the position.

$$
a=(F-(1 / 2) \rho S C d) /\left(m+m_{a}\right)
$$

TABLE II: PARAMETERS OF THE SIMULATION 
The test was conducted in the sway direction. The heave direction was not tested because the effect of fluid parameters in heave direction is small for the simulations described below. Table II shows the parameters of the test bed for the model. Note that $L$ and $n$ in the Table II denote cable length and the number of cable elements, respectively. $n$ is set to six here.

\section{Verification of the Model Using Step Response}

In order to verify the constructed model, a step response test was also conducted using the test bed of the dam inspection system and the simulation. The UWP was initially placed immediately beneath the SWP, and a force of $19.6 \mathrm{~N}$ was applied to the SWP in the $X_{o}$ direction until the $X_{o}$ position of the SWP reached $1.9 \mathrm{~m}$. The Runge-Kutta method was used for the numerical calculation of the simulation, and its time step corresponded to $0.01 \mathrm{~s}$. The experiment of the test bed was conducted at a pool. The force was applied to the SWP by pulling a rope tied to the SWP using a pulley and a weight. The fall distance of the weight corresponded to $1.9 \mathrm{~m}$ (Fig. 6 and Fig. 7). The changing position of the test bed was calculated from a video recorded by the test bed. The video captured the ropes lowered from the surface water at equal intervals, or seam of panels placed on the pool wall, thereby providing distance landmarks for the calculation. The results of the experiments and the simulation are shown in Fig. 8, Fig. 9 and Fig. 10. The horizontal axis denotes the time (in seconds), and the vertical axis denotes the change in the position of the SWP and the UWP. Each graph indicates that the SWP first begins moving, this is followed by the movement of the UWP after a delay, and finally the UWP restores its own position immediately beneath the SWP. Additionally, with respect to the changes in the parameters, an increase in the cable length slows the response of the UWP to the motion of the SWP, and an increase in the weight of the UWP in water increases the speed of the response. The motion of the test bed and the simulation are in good agreement while the external force is applied. However, in the latter half of the simulation the SWP and the UWP are oscillating in opposite phases. This amplitude decreased as the added mass of UWP decreased. It seems to be due to the interaction between the SWP and the UWP, so it is considered that the balance of the added mass of both vehicles is influencing the oscillating phenomenon.

\section{Evaluation of Verticality of the Underwater Platform by Simulation}

First, a simulation is used to evaluate the position difference between the SWP and UWP while the dam inspection system performs an inspection in the water current In the simulation, the water is fixed at the field fixed coordinate system, and the SWP is cruised at a speed identical to that of the water current. This is followed by evaluating the steady state of the position difference.

The simulation result of the position difference for each cable length is shown in Fig. 11. The position difference increases with increases in the flow rate and cable length. Thus, in situations involving a low water current, it is considered that the positioning method of the dam inspection system achieves practical accuracy. In most cases, the dam inspection is performed while the water discharge is stopped. Hence, the water current around the dam facility is considered low. Furthermore, the simulation result when the weight in water and the drag coefficient in the sway direction of the UWP are varied is shown in Fig. 12 and Fig. 13, respectively. These graphs indicate that that the position estimation accuracy of the dam inspection system is enhanced by designing the UWP such that it has a high weight in water and by reducing the fluid drag in the sway direction.

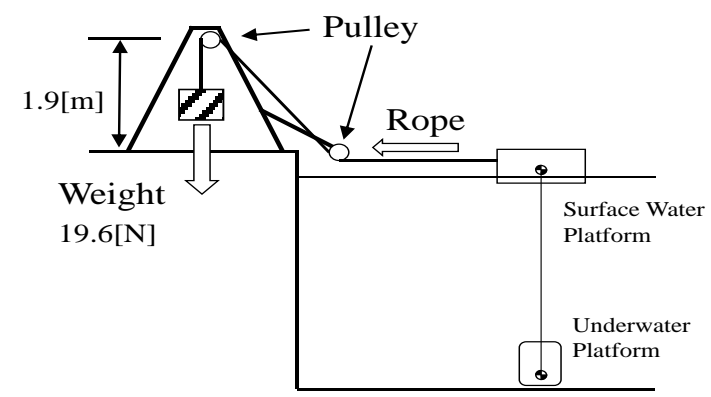

Fig. 6. Configuration of step response experiment.

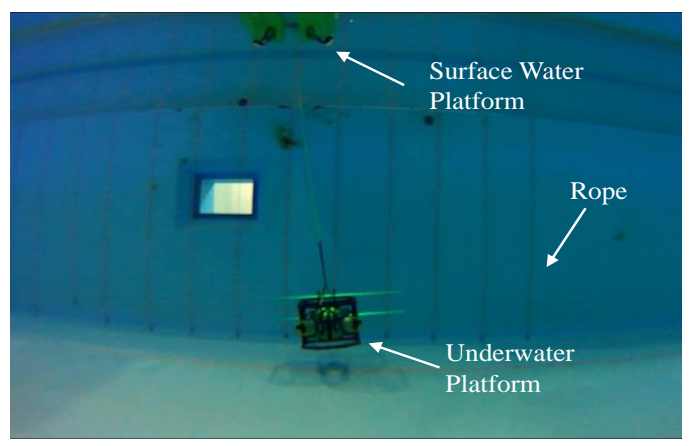

Fig. 7. A landscape while step response experiment.

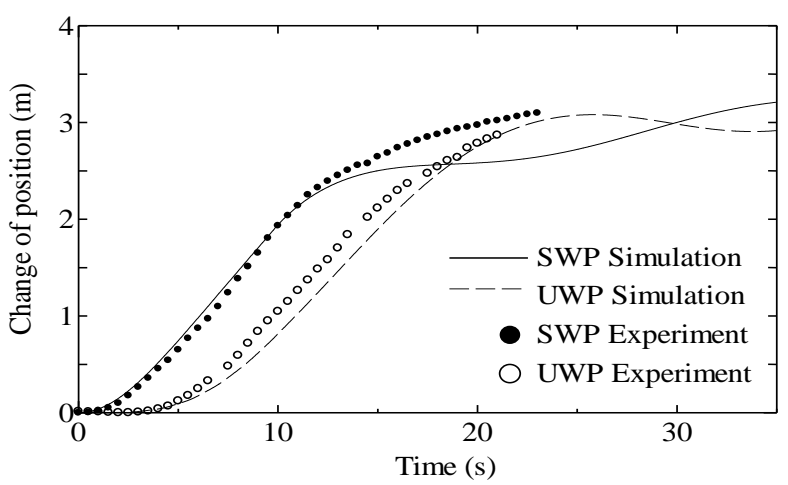

Fig. 8. Cable length $=2.8(\mathrm{~m}), W_{a q}=1.7(\mathrm{~kg})$.

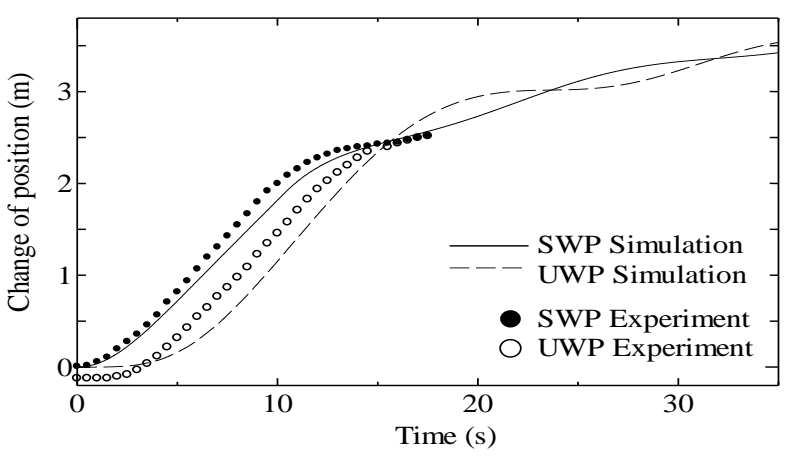

Fig. 9. Cable length $=2.8(\mathrm{~m}), W_{a q}=3.5(\mathrm{~kg})$. 


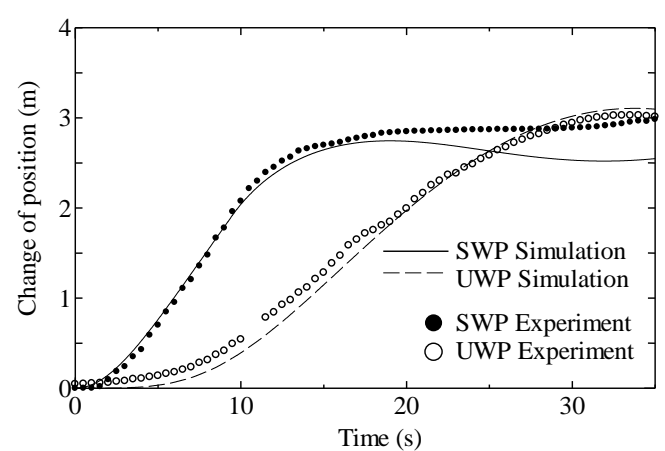

Fig. 10. Cable length $=7.3(\mathrm{~m}), W_{a q}=1.7(\mathrm{~kg})$.

This is followed by evaluating the position difference between the SWP and UWP while the dam inspection system is in motion by using a simulation. The position difference when the dam system moves with only the thruster of the SWP is considered to be identical to the result of the step response experiment. It is necessary for the operator to wait until the UWP restores its own horizontal position to the position immediately beneath the SWP. It is not impractical. Hence, a movement method that uses the thruster of the SWP as well as the thruster of the UWP is used. Additionally, a simple method involved attaching the feed-forward controller that is shown in Fig. 14 independently to the thrusters of both vehicles and using this method to evaluate the position difference between the SWP and UWP while the vehicles are in motion. In the simulation, the step speed at a speed of $0.2 \mathrm{~m} / \mathrm{s}$ in the sway direction is input to the SWP and the UWP from a state at which the UWP is hanged from the SWP and is stationary. Fig. 15 shows the position and velocity of the SWP and the UWP when the cable length corresponds to $20 \mathrm{~m}$. And Fig. 16 shows the position difference for each cable length. The position difference between the SWP and the UWP increases with increases in the cable length, but the difference is small compared to the step response. Although this moving method is simple, it accurately decreases the difference between the SWP and UWP. Additionally, the difference increases with increases in the time. It is considered that the effect of the cable that is ignored in this method is accumulated. Thus, this moving method increases the position estimation error when movement occurs over a long period of time. However, this effect does not potentially pose a problem for the dam inspection system in this study because its purpose relates to the visual inspection of the dam and moves slowly with respect to a high definition video recording. Furthermore, this effect can be reduced by operating the main operation direction of the dam inspection system in the heave direction.

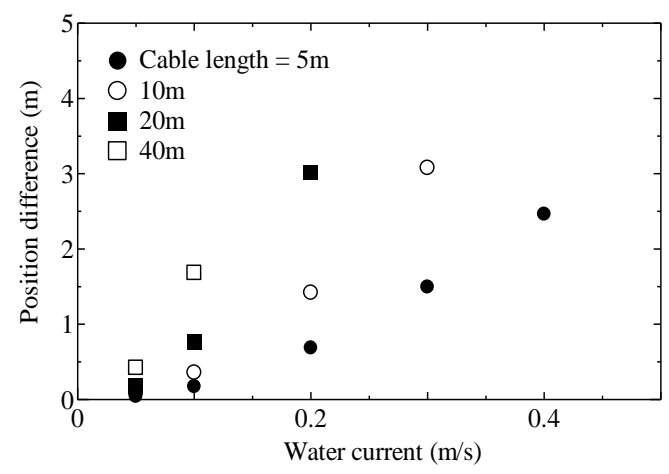

Fig. 11. Position difference in water current.

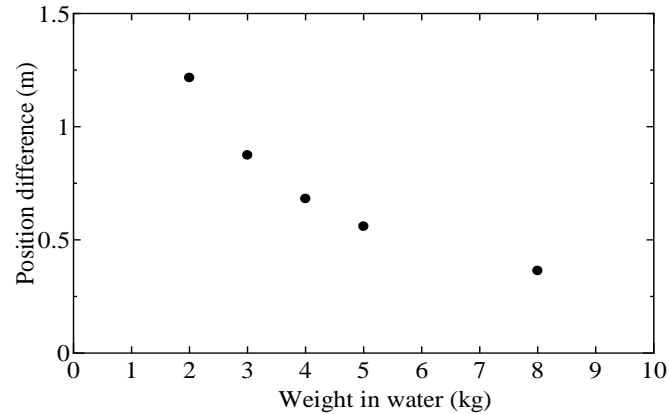

Fig. 12. Position difference when varying the $W_{a q}$. Cable length $=20(\mathrm{~m})$, water current is $0.2(\mathrm{~m} / \mathrm{s})$.

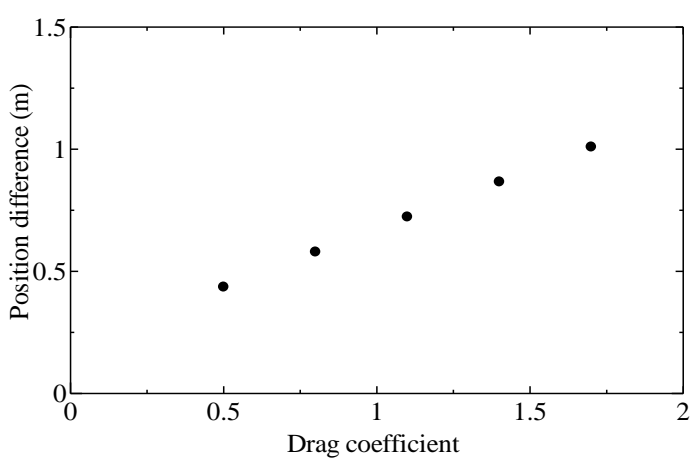

Fig. 13. Position difference when varying the $C d_{x}$ of the UWP. Cable length $=20(\mathrm{~m})$, water current is $0.2(\mathrm{~m} / \mathrm{s})$.

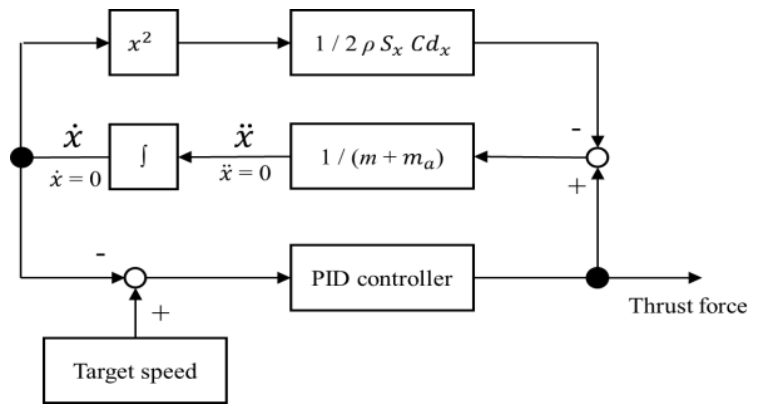

Fig. 14. Attached feed-forward controller.

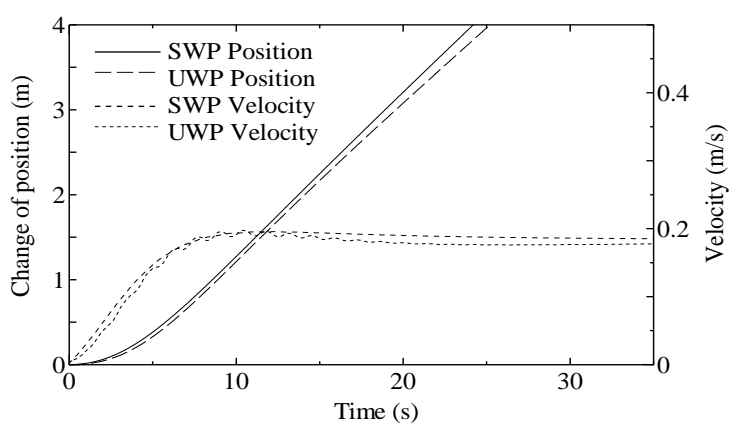

Fig. 15. Positon and velocity of the vehicles moving by feed-forward control.

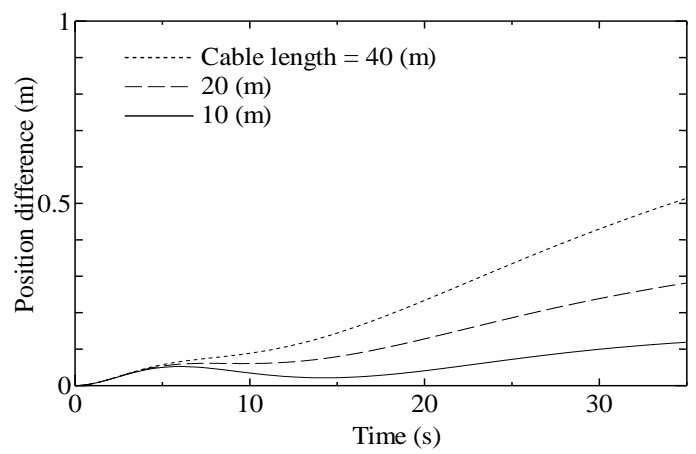

Fig. 16. Position difference between the SWP and the UWP when moving by feed-forward control. 


\section{EXPERIMENT}

The pool testing and the demonstration of inspection at the actual dam were conducted using the developed test bed of the dam inspection system. The position difference between the SWP and UWP is evaluated by the data obtained during the testing and the demonstration.

\section{A. Pool Testing}

The position difference while the test bed is in motion is evaluated by the data obtained from the pool testing. Fig. 16 shows images corresponding to the time-lapse when the test bed moves-in the left direction along the pool wall. The front of the test bed faces the surface of the pool wall. The depth of the UWP corresponds to $4 \mathrm{~m}$. The upper images correspond to those obtained from the SWP, and the lower images correspond to those obtained from the UWP. The vertical orange lines denotes the ropes that are attached on the pool wall as the marker. The thrusts of the SWP and the UWP were independently controlled by the feed-forward control method, and the speed command was based on the move distance error between the desired move distance which is set to $0.82 \mathrm{~m}$ and the imaginary move distance that is calculated from the thrust force. The image on the right side shows the state at which the test bed is in complete side motion. The
SWP and the UWP were positioned at the front of the same rope. This indicated that the UWP was positioned beneath the SWP. In a manner similar to the simulation, it was confirmed that the method that used the thrusters of both vehicles when the dam inspection system moved decreased the position difference between the vehicles.

\section{B. Inspection Demonstration at the Actual Dam}

The inspection demonstration at the actual dam using the dam inspection system was conducted at the Yasaka dam, Japan [7]. The dam is a concrete gravity dam that was constructed in 1921 with a capacity of $112,000,000 \mathrm{~m}^{3}$ and with a maximum wall height of $120 \mathrm{~m}$. The test bed was deployed on the dam lake, and the human interface unit was set on the boat. The operator operated the system from the boat. The measurements for the period of the study indicated a maximum sustained wind speed of $8 \mathrm{~m} / \mathrm{s}$ and a maximum instantaneous wind speed of $18 \mathrm{~m} / \mathrm{s}$. The test bed did not cruise freely due to this the wind. Hence, the SWP was kept at a constant point at the surface of the dam wall, and the vertical inspection was conducted beneath the SWP. The inspection target corresponded to the seam of the concrete and the conduit gate. A portion of three seams was inspected, and the total length corresponded to $85 \mathrm{~m}$.

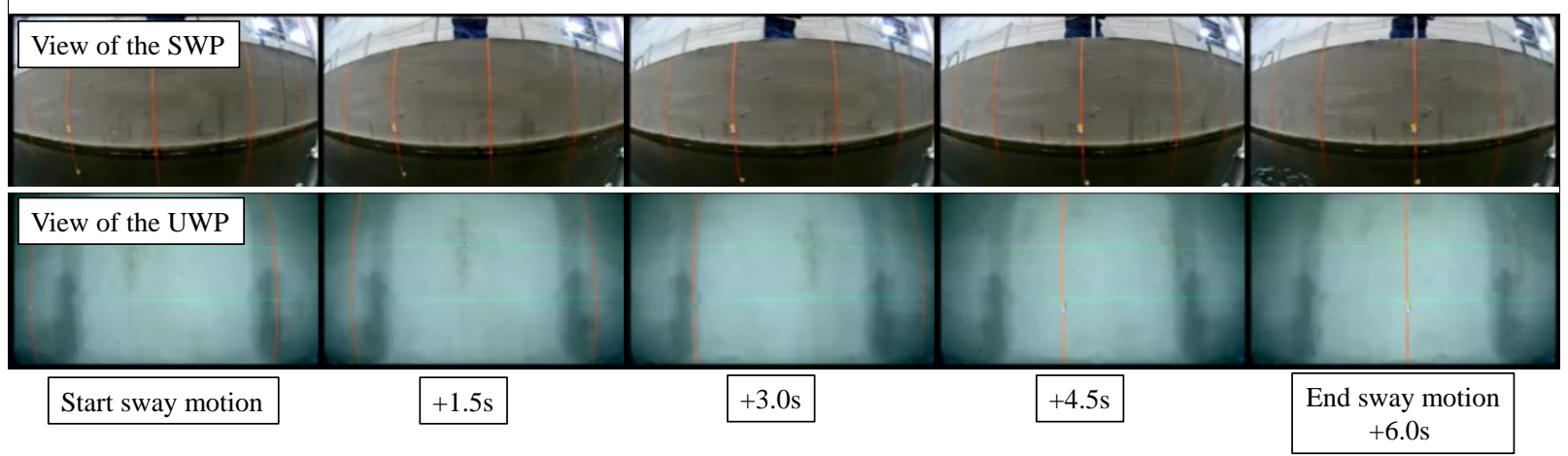

Fig. 17. Time-lapse images when the test bed moving left-sway direction by feed-forward control.

A part of the obtained image is shown in Fig. 18. The SWP was placed in front of the seam when the seam was inspected. The UWP was in descent or ascent along the seam. The guide arm that was attached on both sides of the UWP was used while inspecting the seam. The arm contacted the dam wall by a forward thrust operation. This mechanical contact kept the relative position between the UWP and the dam wall steady and enabled in obtaining a precise image. The arms corresponded to equipped rollers that did not disturb the heave motion of the UWP. The effect of the UWP position immediately beneath the SWP was also confirmed in a real environment. It was easy to position the UWP in front of the concrete seam.

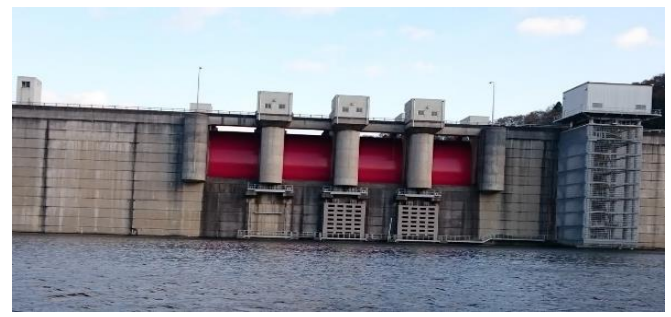

Fig. 18. Yasaka dam.

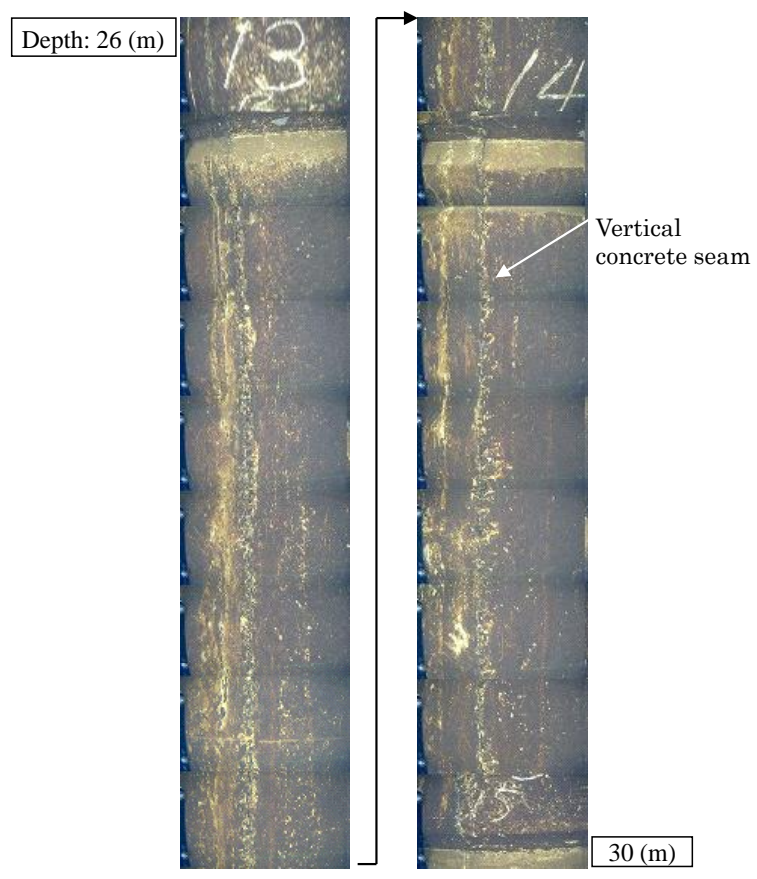

Fig. 19. A part of the image of the concrete seam obtained from demonstration inspection. 


\section{CONCLUSION}

This study reports on the evaluation results of a positioning method of the dam inspection system. The dynamics model is constructed and verified to compare the motion between the simulation and the test bed. This model is used to evaluate the difference in position between the SWP and the UWP when the dam inspection system is in motion and performs an inspection in a water current. The results indicate that the position difference, when the dam inspection system is in motion, decreases due to the motion method that uses both thrusters of the vehicles with a feed-forward control. Additionally, the results indicated that the accuracy of the position estimated by the dam inspection system in a low speed water current is considered to be practical. A pool test experiment was conducted, and it confirmed that the moving method that used the thrusters of both vehicles is also effective in the pool test using the test bed. The findings also confirmed the effect in which the UWP maintained its own horizontal position beneath the SWP in real environment. In a future study, we are going to develop automatic inspection method of proposed system for dam wall. In addition, in order to improve the practicality in the real environment, we are also going to advance performance of the test bed such as maneuverability.

\section{ACKNOWLEDGMENT}

This research is promoted by the consortium consist of Q.I incorporated, National Institute of Advanced Industrial Science and Technology (AIST), and Hitachi, Ltd.

\section{REFERENCES}

[1] White paper on land, infrastructure, transport and tourism in Japan 2013. Ministry of Land, Infrastructure, Transport and Tourism, Japan. [Online]. $28-30 . \quad$ pp. Available: http://www.mlit.go.jp/common/001063075.pdf.

[2] P. Ridao, M. Carreras, D. Ribas, and R. Garcia, "Visual inspection of hydroelectric dams using an autonomous underwater vehicle," $J$. of Field Robotics, vol. 27, no. 6, pp. 759-778, November/December 2010.

[3] N. A. Cruz, A. C. Matos, R. M. Almeida, B. M. Ferreira, and N. Abreu, "TriMARES - A hybrid AUV/ROV for dam inspection," OCEANS 2011 MTS/IEEE, pp. 1-7,pp. 19-22 Sept. 2011.

[4] S. Shimono, S. Toyama, U. Nishizawa, O. Matsubara, S. Kato, and H. Arisumi, "Development of underwater inspection system for dam inspection: Concept and evaluation of simplified dynamics model," OCEANS 2015 MTS/IEEE, pp. 1-6, pp. 19-22, Oct. 2015.

[5] B. Buckham, M. Nahon, M. Seto, X. Zhao, and C. Lambert, "Dynamics and control of a towed underwater vehicle system, part I: Model development," Ocean Engineering, vol. 30, no. 4, pp. 453-470, March. 2003.

[6] F. R. Driscoll, R. G. Lueck, and M. Nahon, "Development and validation of a lumped-mass dynamics model of a deep-sea ROV system," Applied Ocean Research, vol. 22, no. 3, pp. 169-182, June. 2000 .

[7] S. Shimono, S. Toyama, and U. Nishizawa, "Development of underwater inspection system for dam inspection: Results of field tests," OCEANS 2016 MTS/IEEE, pp. 1-4, pp. 19-23, Sept. 2016.

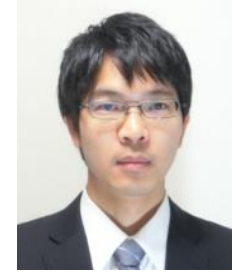

Soji Shimono was born in Tokyo, Japan, in 1988. He received his B.E., M.E., in mechanical engineering from Tokai university, Japan in 2009, 2011, respectively. He is 2nd year student in the doctoral program of Tokyo University of Agriculture and Technology. Since 2012, He is an engineer of Q.I incorporated. His primary research interests include robotics. He is a member of the Marine Technology Society.

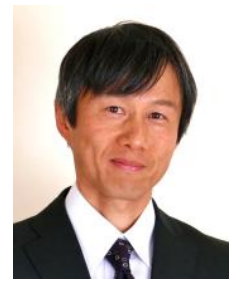

Hitoshi Arisumi received the B.E., M.E., and Ph.D degrees in mechanical engineering science from Tokyo Institute of Technology, Tokyo, Japan, in 1991, 1993, and 2003, respectively. In 1993, he joined the Mechanical Engineering Laboratory, Ministry of International Trade and Industry, which is currently National Institute of Advanced Industrial Science and Technology (AIST), Tsukuba, Japan. From June 2003 to June 2004, he was with the Interdepartmental Research Center, "E. Piaggio," University of Pisa, Pisa, Italy, as a Visiting Scholar. He is currently a senior research scientist of Field Robotics Research Group, Intelligent System Institute, AIST. His current research interests include autonomous control of mobile robots, humanoids, and dynamic manipulation. Dr. Arisumi is a member of the Robotics Society of Japan (RSJ) and the Japan Society of Mechanical Engineers (JSME).

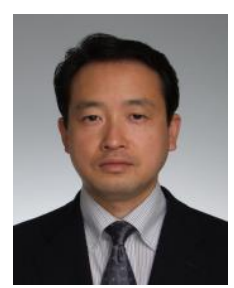

Shin Kato was born in Chiba, Japan, in 1967. He received his B.E., M.E., and Dr. Eng. degrees in electrical engineering from Meiji University, Japan in 1989, 1991, and 1994, respectively. Since 1994, He was a domestic research fellow of Japan Science and Technology Corporation. In 1997, He joined Mechanical Engineering Laboratory, Ministry of International Trade and Industry, currently National Institute of Advanced Industrial Science and Technology (AIST). Now, he is a group leader at Intelligent Systems Research Institute, AIST. His research interests are in the fields of ITS (Intelligent Transport Systems), AVCSS (Advanced Vehicle Control and Safety Systems), machine vision, sensing, communications, navigation, HMI (Human Machine Interface) and control for intelligent vehicles and robots. $\mathrm{He}$ is a member of the Society of Automotive Engineers of Japan, the Robotics Society of Japan, the Japan Society of Mechanical Engineers, and so on.

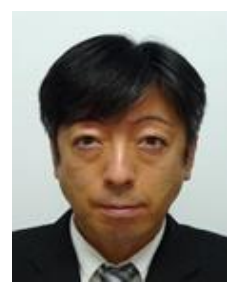

Cichi Nishizawa received his Ph.D. degree in Engineering from Tokyo University of Agriculture and Technology, Tokyo, Japan, in 2008. Now he works at Tokyo University of Agriculture and Technology. His current research interests include robotics, aerospace engineering, and welfare technology.

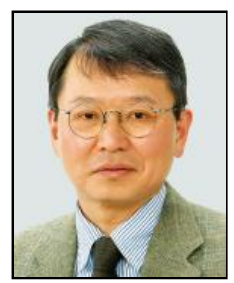

Shigeki Toyama received his $\mathrm{Ph} . \mathrm{D}$. degree in engineering from University of Tokyo, Tokyo, Japan, in 1981. Now he works at Tokyo University of Agriculture and Technology. His current research interests include robotics, actuator, and welfare technology. 